\title{
Quantum states of a particle in a box via unilateral Fourier transform
}

\author{
Douglas W. Vieira*1, Antonio S. de Castro ${ }^{10}$ \\ ${ }^{1}$ Universidade Estadual Paulista "Júlio de Mesquita Filho", Departamento de Física, Guaratinguetá, SP, Brasil
}

Received on April 20, 2020. Accepted on May 14, 2020.

\begin{abstract}
The quantum problem of stationary states of a particle in a box is revisited by means of the unilateral Fourier transform. Homogeneous Dirichlet boundary conditions demand a finite Fourier sine transform which is actually the Fourier sine series.
\end{abstract}

Keywords: Particle in a box, Infinite square-well potential, Unilateral Fourier transform.

\section{Introduction}

In quantum theory, a particle confined by impenetrable walls is usually called a particle in box. For onedimensional cases that kind of system is modeled by an infinite square-well potential. This is one of the easiest problems in quantum mechanics exhibiting many characteristics of the quantum physics and for this reason it appears in a plethora of introductory textbooks on quantum mechanics (see, e.g. [1]- [12]). Although it is not a realistic system, it serves as an idealization of complex systems occurring in the nature and, in some circumstances, reflects the properties of certain real systems. Unremarkably, the possible nonrelativistic bound-state solutions of a particle in a one-dimensional box are found by a straight and short resolution of the time-independent Schrödinger equation by imposing the continuity of the eigenfunctions on the confining walls. By contrast, in a recent paper diffused in the literature, the quantum problem of a particle in an infinite square-well potential was claimed to be solved via Laplace transform 13 . While emphatically refuted due to an erroneous inversion of the Laplace transform [14], Ref. 13 awakens interest in applying over a finite interval other kinds of integral transforms usually defined over an infinite or a semi-infinite range of integration.

In this work we approach the quantum problem of a particle in an infinite square-well potential with the unilateral Fourier transform. Ordinarily the unilateral Fourier transform is a useful tool for absolutely integrable functions defined over a semi-infinite interval depending on the homogeneous Dirichlet or the homogeneous Neumann boundary conditions at the origin. The way we are going to approach this problem, though, results in a finite Fourier sine transform. That kind of finite unilateral Fourier transform, and its close connection with Fourier series, can be of interest of teachers and stu-

*Correspondence email address: antonio.castro@unesp.br dents of mathematical methods applied to physics and quantum mechanics of undergraduate courses.

\section{Unilateral Fourier transform}

The Fourier sine and cosine transforms of $f(x)$ are denoted by $\mathcal{F}_{s}\{f(x)\}=F_{s}(k)$ and $\mathcal{F}_{c}\{f(x)\}=F_{c}(k)$, respectively, and are defined by the integrals (see, e.g. [15]- 17])

$$
\begin{aligned}
& F_{s}(k)=\mathcal{F}_{s}\{f(x)\}=\sqrt{\frac{2}{\pi}} \int_{0}^{\infty} d x f(x) \sin k x, \\
& F_{c}(k)=\mathcal{F}_{c}\{f(x)\}=\sqrt{\frac{2}{\pi}} \int_{0}^{\infty} d x f(x) \cos k x,
\end{aligned}
$$

where $k>=0$. The original function $f(x)$, based on certain conditions, can be retrieved by the inverse unilateral Fourier transforms $\mathcal{F}_{s}^{-1}\left\{F_{s}(k)\right\}$ and $\mathcal{F}_{c}^{-1}\left\{F_{c}(k)\right\}$ expressed as

$$
f(x)=\sqrt{\frac{2}{\pi}} \int_{0}^{\infty} d k F_{s}(k) \sin k x,
$$

and

$$
f(x)=\sqrt{\frac{2}{\pi}} \int_{0}^{\infty} d k F_{c}(k) \cos k x .
$$

Sufficient conditions for the existence of the above integrals are ensured if $f(x), F_{s}(k)$ and $F_{c}(k)$ are absolutely integrable. The choice of sine or cosine transform is decided by the homogeneous boundary conditions at the origin: Dirichlet condition $\left(\left.f(x)\right|_{x=0}=0\right)$ or Neumann condition $\left(d f(x) /\left.d x\right|_{x=0}=0\right)$.

\section{The particle in a box}

The time-independent Schrödinger equation (for the stationary states) reads

$$
\left[-\frac{\hbar^{2}}{2 m} \frac{d^{2}}{d x^{2}}+V(x)\right] \psi_{E}(x)=E \psi_{E}(x) .
$$


The quantity $\left|\psi_{E}(x)\right|^{2}$ is the position probability density, meaning that $\left|\psi_{E}(x)\right|^{2} d x$ is the probability of finding the particle in the region $d x$ about its point $x$. Then,

$$
\int_{-\infty}^{+\infty} d x\left|\psi_{E}(x)\right|^{2}=1 .
$$

The desired solution of this eigenvalue problem is the characteristic pair $\left(E, \psi_{E}\right)$ with $E \in \mathbb{R}$ and $\psi_{E}(x)$ is single valued, finite and continuous everywhere.

The infinite square-well potential

$$
V(x)=\left\{\begin{array}{l}
0, \quad 0 \leq x \leq L \\
\infty, \quad x<0 \text { and } \quad x>L
\end{array}\right.
$$

emulates a particle constrained to move between two impenetrable walls at a distance $L$ in such a way that one can write

$$
\left(\frac{\hbar^{2}}{2 m} \frac{d^{2}}{d x^{2}}+E\right) \psi_{E}(x)=0, \quad 0 \leq x \leq L,
$$

and

$$
\psi_{E}(x)=0, \quad x<0 \text { and } x>L .
$$

Continuity of the eigenfunction at the walls requires $\psi_{E}(0)=\psi_{E}(L)=0$. Therefore, the eigenfunction $\psi_{E}(x)$ can be compactly written as

$$
\psi_{E}(x)=\theta(x) \theta(L-x) f_{E}(x),
$$

where $\theta(x)$ is is the step function

$$
\theta(x)= \begin{cases}1, & x>0 \\ 0, & x<0\end{cases}
$$

and $f_{E}(x)$ satisfies the equation

$$
\left(\frac{d^{2}}{d x^{2}}+k^{2}\right) f_{E}(x)=0, \quad 0 \leq x \leq L,
$$

subject to the homogeneous Dirichlet boundary conditions $f_{E}(0)=f_{E}(L)=0$, and

$$
\int_{0}^{L} d x\left|f_{E}(x)\right|^{2}=1 .
$$

\section{The solution of the problem}

To begin with, we discard the Fourier cosine transform due to the homogeneous Dirichlet boundary condition at the origin. Rather, we suppose that $f_{E}(x)$ can be expressed by a Fourier sine transform as

$$
f_{E}(x)=\int_{0}^{\infty} d k F^{(E)}(k) \sin k x .
$$

Furthermore, the remaining homogeneous Dirichlet boundary condition at $x=L$ enforces that $k$ is restricted to discrete values

$$
k=\frac{n \pi}{L}, \quad n=1,2,3, \ldots
$$

so that the function $F^{(E)}(k)$ can be regarded as an infinite set of numbers $F_{n}^{(E)}$. Moreover, instead of an integral over the continuous variable $k$, we have a sum over $n$ :

$$
f_{E}(x)=\sum_{n=1}^{\infty} F_{n}^{(E)} \sin \frac{n \pi x}{L} .
$$

The alert reader can see that 16 is just a Fourier sine series as has been already suggested in Ref. [14]. Substitution of this Fourier sine series into Eq. 12 furnishes

$$
\sum_{n=1}^{\infty}\left(\frac{\hbar^{2} \pi^{2} n^{2}}{2 m L^{2}}-E\right) F_{n}^{(E)} \sin \frac{n \pi x}{L}=0 .
$$

Multiplying this series by

$$
\sin \frac{\tilde{n} \pi x}{L}, \quad \widetilde{n}=1,2,3, \ldots,
$$

and integrating from 0 to $L$, we find

$$
\sum_{n=1}^{\infty}\left(\frac{\hbar^{2} \pi^{2} n^{2}}{2 m L^{2}}-E\right) F_{n}^{(E)} \int_{0}^{L} d x \sin \frac{n \pi x}{L} \sin \frac{\tilde{n} \pi x}{L}=0 .
$$

Taking advantage of the orthonormality relation

$$
\int_{0}^{L} d x \sin \frac{n \pi x}{L} \sin \frac{\tilde{n} \pi x}{L}=\frac{L}{2} \delta_{n \widetilde{n}}
$$

where $\delta_{n \tilde{n}}$ is the Kronecker delta symbol

$$
\delta_{n \tilde{n}}=\left\{\begin{array}{cc}
1, & \widetilde{n}=n \\
0, & \widetilde{n} \neq n
\end{array}\right.
$$

we find

$$
\sum_{n=1}^{\infty}\left(\frac{\hbar^{2} \pi^{2} n^{2}}{2 m L^{2}}-E\right) F_{n}^{(E)} \delta_{n \tilde{n}}=0
$$

in such a way that the Kronecker delta symbol kills every term in the sum except the one for which $n=\widetilde{n}$. Then, the left-hand side of 22 reduces to one term:

$$
\left(\frac{\hbar^{2} \pi^{2} n^{2}}{2 m L^{2}}-E\right) F_{n}^{(E)}=0 .
$$

Taking one and only one $F_{n}^{(E)} \neq 0$ we find

$$
f_{n}(x)=F_{n}^{(n)} \sin \frac{n \pi x}{L},
$$

with

$$
E_{n}=\frac{\hbar^{2} \pi^{2} n^{2}}{2 m L^{2}}
$$

and the eigenfunctions are finally expressed as

$$
\psi_{n}(x)=\theta(x) \theta(L-x) \sqrt{\frac{2}{L}} \sin \frac{n \pi x}{L},
$$

where $F_{n}^{(n)}=\sqrt{2 / L}$ was determined by $\sqrt{13}$. This characteristic par $\left(E_{n}, \psi_{n}\right)$, given by 25 and $(26)$, is in agreement with that one found by usual methods. 


\section{Final remarks}

We have shown that the stationary states of the particle in a box via unilateral Fourier transform can be found with simplicity because it is a tool that favors compliance with boundary conditions from the start. Regarding the Laplace transform used in Ref. 13.

$$
\mathcal{L}\left\{\psi_{E}(x)\right\}=\int_{0}^{L} d x e^{-s x} f_{E}(x),
$$

it was shown in Ref. [14 that

$$
\begin{aligned}
\mathcal{L}\left\{\frac{d^{2} \psi_{E}(x)}{d x^{2}}\right\} & =s^{2} \mathcal{L}\left\{\psi_{E}(x)\right\}-\left.\frac{d f_{E}(x)}{d x}\right|_{x=0} \\
& +\left.e^{-s L} \frac{d f_{E}(x)}{d x}\right|_{x=L}
\end{aligned}
$$

so that all the inconvenience of the finite Laplace transform is due to the border term proportional to $e^{-s L}$ that vanishes only when $\operatorname{Re} s>0$ and $L \rightarrow \infty$. On the other hand, it can be shown that

$$
\mathcal{F}_{s}\left\{\frac{d^{2} \psi_{E}(x)}{d x^{2}}\right\}=-k^{2} \mathcal{F}_{s}\left\{\psi_{E}(x)\right\},
$$

without border terms in such a way that

$$
\mathcal{F}_{s}^{(n)}\left\{\psi_{E}(x)\right\}=\sqrt{\frac{2}{\pi}} \int_{0}^{L} d x f_{E}(x) \sin \frac{n \pi x}{L},
$$

furnishes

$$
\left(\frac{\hbar^{2} \pi^{2} n^{2}}{2 m L^{2}}-E\right) \mathcal{F}_{s}^{(n)}\left\{\psi_{E}(x)\right\}=0 .
$$

As a matter of fact, the homogeneous Dirichlet boundary condition at $x=L$ has allowed to change by reversal the usual transition from a Fourier series to a Fourier transform (see, e.g. 15- [16]). The problem of a particle in a box symmetric about $x=0$, and the related Fourier sine transform and Fourier cosine transform, is left for the readers.

\section{Acknowledgement}

Grant 09126/2019-3, Conselho Nacional de Desenvolvimento Científico e Tecnológico (CNPq), Brazil.

\section{References}

[1] D.J. Griffiths, Introduction to Quantum Mechanics (Prentice Hall, New Jersey, 1955).

[2] A. Messiah, Mecanique Quantique (Dunod, Paris, 1964), v. 1.

[3] A.S. Davydov, Quantum Mechanics (Pergamon Press, Oxford, 1965).

[4] L.I. Schiff, Quantum Mechanics (McGraw-Hill, New York, 1968).
[5] G. Baym, Lectures in Quantum Mechanics (Benjamin, New York, 1969).

[6] E. Merzbacher, Quantum Mechanics (Wiley, New York, 1970).

[7] S. Flügge, Practical Quantum Mechanics (SpringerVerlag, Berlin, 1971), v. 1.

[8] R. Eisberg and R. Resnick, Quantum Physics of Atoms, Molecules, Solids, Nuclei and Particles (Wiley, New York, 1974).

[9] C. Cohen-Tannoudji, D. Bernard and F. Laloë, Quantum Mechanics (Hermann, Paris, 1977), v. 1.

[10] W. Greiner, Quantum Mechanics: An Introduction (Springer-Verlag, Berlin, 1989).

[11] R. Shankar, Principles of Quantum Mechanics (Plenum Press, New York, 1994).

[12] R.W. Robinett, Quantum Mechanics (Oxford University Press, Oxford, 2006), 2nd ed.

[13] R. Gupta, R. Gupta and D. Verma, IJITEE 8, 6 (2019).

[14] A.S. Castro, Rev. Bras. Ens. Fis. 42, e20200079 (2020).

[15] E. Butkov, Mathematical Physics (Addison-Wesley, Reading, 1968).

[16] G.B. Arfken and H.J. Weber, Mathematical Methods for Physicists (Harcourt/Academic Press, San Diego, 1996), 5th ed.

[17] I.S. Gradshteyn and I.M. Ryzhik, Table of Integrals, Series, and Products (Academic, New York, 2007), 7th ed. 